\title{
GROWTH RATES FOR GEOMETRIC COMPLEXITIES AND COUNTING FUNCTIONS IN POLYGONAL BILLIARDS
}

\author{
EUGENE GUTKIN AND MICHAL RAMS
}

\begin{abstract}
We introduce a new method for estimating the growth of various quantities arising in dynamical systems. We apply our method to polygonal billiards on surfaces of constant curvature. For instance, we obtain power bounds of degree two plus epsilon for billiard orbits between almost all pairs of points in a planar polygon.
\end{abstract}

\section{INTRODUCTION AND OVERVIEW}

Complexity of a dynamical system is measured with respect to a coding of its orbits. The coding, in turn, is determined by partitioning the phase space of the system into elementary pieces. For dynamical systems with singularities, such as polygonal billiards, connected components in the complement to the singular set yield a natural partition. Convexity of its atoms with respect to the geodesic structure in the phase space imposed by geometric optics, is crucial in the study of billiard complexity [7].

In the present study, as well as in [7, $P$ is a geodesic polygon in a surface of constant curvature. Let, for concreteness, $P$ be a planar polygon. We denote by $f_{P}(n)$ the number of words of length $n$ generated by coding billiard orbits by visited domains of regularity. When $P$ is simply connected, this coincides with the coding by sides in $P$. It is known that $f_{P}(n)$ is subexponential in $n$ [3, 6], and for general $P$ no better bound is known. If $P$ is a rational polygon (i. e., its angles are commensurable with $\pi$ [4]), $f_{P}(n)=O\left(n^{3}\right)$ [1, 17]. The current conjecture is that for any planar polygon $f_{P}(n)=O\left(n^{d}\right)[5]$.

In order to advance the understanding of billiard complexity, we introduce the notion of partial complexities. Let $\Psi$ be the phase space, and let $\mathcal{P}$ be the defining partition. Iterating the dynamics we obtain an increasing tower $\mathcal{P}_{n}$ of partitions; the full complexity is $f(n)=$

Date: October 31, 2018.

Key words and phrases. Geodesic polygon, billiard map, billiard flow, complexity, counting functions, unfolding of orbits, covering space, exponential map. 
$\left|\mathcal{P}_{n}\right|$. If $R \subset \Phi$, let $\mathcal{P}_{n}(R)$ be the induced tower of its partitions. The partial complexity based on $R$ is $f_{R}(n)=\left|\mathcal{P}_{n}(R)\right|$. Particular partial complexities have been studied earlier. For instance, in [8] we obtained polynomial bounds on direction complexity, which is one of the partial complexities investigated here.

In this work we introduce a new general approach to estimating partial complexities. The setting is as follows. There is a family of subsets $R_{\theta}$ foliating the phase space. Let $f_{\theta}(n)$ be the partial complexity with base $R_{\theta}$. Let $g_{\theta}(n)$ be the counting function for singular billiard orbits starting from $R_{\theta}$. Under appropriate assumptions, $f_{\theta}(n)$ and $g_{\theta}(n)$ have the same growth, as $n \rightarrow \infty$. See section 4 .

Let $\theta \in \Theta$, the parameter space. Suppose that we bound the average counting function $G(n)=\int_{\Theta} g_{\theta}(n)$. Tchebysheff inequality and the zero-one law yield bounds for individual $g_{\theta}(n)$ valid for almost all $\theta \in \Theta$. See section 2. Combined with preceding remarks, these yield estimates on partial complexities for almost all values of the parameter.

This is the general scheme for our approach to partial complexities. This work implements this scheme for polygonal billiards. We will now describe the contents of the paper in more detail.

In section 1 we investigate counting functions and their averages. We establish the relevant framework in sufficient generality, with the view towards a broad range of geometric-dynamic applications. The main results are Propositions 1 and 2 respectively. These yield geometric formulas for averages of counting functions which are valid under mild assumptions of transversality type.

Section 2 is analytic, and also quite general. The setting is as follows. There is a family of positive functions, $g_{\theta}(p)$, of positive argument ( $p \in \mathbb{N}$ and $p \in \mathbb{R}_{+}$in the discrete and continuous cases respectively), depending on parameter $\theta \in \Theta$. Set $G(p)=\int_{\Theta} g_{\theta}(p) d \theta$. From upper bounds on $G(p)$ we derive estimates on individual $g_{\theta}(p)$; they are valid for almost all $\theta$. Precise formulations depend on the details of the situation. See Propositions 3 and 4 .

Section 3 sets the stage for applications to billiard dynamics. Our billiard table is a geodesic polygon, $P$, in a simply connected surface of constant curvature. There are two versions of billiard dynamics: the billiard flow and the billiard map. In our discussion of partial complexities, it is convenient to treat them separately. Accordingly, section 3 consists of several subsections; each subsection deals with a particular partial complexity for a particular kind of billiard dynamics.

We use two geometric parameters for partial complexities: the direction and the position. The direction complexity tells us how the set of 
phase points starting in the same direction splits after bouncing off of the sides of $P$. The direction complexity is defined for planar polygons. The position complexity tells us about the splitting of beams of billiard orbits emanating from a point of $P$. It is defined in all cases.

In each of the subsections of section 3 we define a counting function and check the assumptions of section 1, then we evaluate the integral over the parameter space, i. e., we compute the average counting functions. It turns out that they have geometric meanings. Here is a sample of results from section 3. Let $G_{P}(l)$ be the average position counting function for the billiard flow in a geodesic polygon $P$. For planar polygons we have $G_{P}(l)=c_{0}(P) l^{2}$. See Corollary 2 in section 3.2 . For polygons in $\mathbb{S}^{2}$ we have $G_{P}(l)=c_{+}(P) l+c_{+}^{\prime}(P) f(l)$ where $f$ is a universal periodic function. See Corollary 3 in section 3.4 . For polygons in $\mathbb{H}^{2}$ we have $G_{P}(l)=c_{-}(P) \cosh l$. See Corollary 4 in section 3.5, The coefficients in these formulas depend on how many corners $P$ has and on the number of obstacles in its interior.

Section 4, again, is quite general. In this section we obtain relationships between partial complexities with one-dimensional base sets and counting functions. The main result of this section is Proposition 5 , It says that if the bases are one-dimensional, then the difference between the partial complexity and the counting function is bounded, as time goes to infinity. Other assumptions on the base have to do with convexity in the phase space. The framework of this section is that of piecewise convex transformations [7].

In section 5 we specialize again to polygonal billiards. Combining the material of preceding sections, we obtain bounds on the position and direction complexities for the billard flow and the billiard map. Here is a sample of our results. Let $P$ be a euclidean polygon. Let $\theta \in S^{1}$ (resp. $z \in P$ ) be any direction (resp. position). Let $f d_{\theta}(n)\left(\operatorname{resp} . h_{z}(l)\right)$ be the direction complexity for the billiard map (resp. position complexity for the billiard flow). Then for almost all directions $\theta$ (resp. for almost all positions $z$ ) we have $f d_{\theta}(n)=O\left(n^{1+\varepsilon}\right)\left(\operatorname{resp} . \quad h_{z}(l)=O\left(l^{2+\varepsilon}\right)\right)$, where $\varepsilon>0$ is arbitrary. See Corollary 6 and Corollary 8 , Let now $P$ be a spherical polygon, and let $h_{z}(l)$ be the position complexity for the billiard flow in $P$. Then for almost every $z \in P$ there is a $C=C(z)$ and arbitrarily large $l$ such that $h_{z}(l) \leq C l$. See Corollary 10. For any $\varepsilon>0$ and almost every $z \in P$ we have $h_{z}(l)=O\left(l^{1+\varepsilon}\right)$. See Corollary 11 .

In the study of polygonal billiards the device of unfolding billiard orbits is indispensable [4]. If $P \subset M$, and $\beta$ is a billiard orbit in $P$, its unfolding is a geodesic in $M$. Several arguments in section 3 use 
the technique of lifting billiard orbits to the universal covering space of $P 1$ This notion was not written up in the billiard literature. In our Appendix section 6 we present the relevant definitions and propositions. Proposition 6 puts forward the main property of the universal covering space of a geodesic polygon. It relates the unfoldings and the liftings of billiard orbits. The proofs in section 3 use Corollary 13 of Proposition 6 , which deals with the pullbacks of lebesgue measures under unfoldings.

In order to put our results into perspective, we will now briefly survey the literature on billiard complexities. The subexponential growth of (full) billiard complexity for arbitrary euclidean polygons is established in [3] and [6]. Both proofs are indirect, in that they do not yield explicit subexponential bounds. On the other hand, for rational euclidean polygons the complexity is cubic. This is contained in [1] for convex and in [7] for all rational polygons. The arguments in [1] and [7. rely on a theorem in [11; it says that the number of billiard orbits between any pair of corners in a rational polygon grows quadratically in length. From our viewpoint, this is a statement about the position counting functions $g_{z}(l)$. It says that $g_{z}(l)=O\left(l^{2}\right)$ if $P \subset \mathbb{R}^{2}$ is rational and $z \in P$ is a corner. By comparison, our Corollary 8 and Proposition 5 yield that $g_{z}(l)=O\left(l^{2+\varepsilon}\right)$ for any $\varepsilon>0$ and almost all $z \in P$ where $P \subset \mathbb{R}^{2}$ is an arbitrary polygon. The directional complexity $f d_{\theta}(n)$ has been studied in [8] and [9]. The work [9] concerns the directional complexity for the billiard in a rational, planar polygon $P$. Assume that $P$ is convex. Then [9] derives an explicit formula for $f d_{\theta}(n)$, valid for minimal directions $\theta$. (The set of nonminimal directions is countable.) By this formula, $f d_{\theta}(n)=O(n)$. On the other hand, 8] shows that $f d_{\theta}(n)=O\left(n^{d}\right)$ for any $P \subset \mathbb{R}^{2}$ and an arbitrary $\theta$. The degree $d$ in the bound does not depend on $\theta$. Our Corollary 6 estimates the complexity $f d_{\theta}(n)$ for an arbitrary polygon $P \subset \mathbb{R}^{2}$. It says that $f d_{\theta}(n)=O\left(n^{1+\varepsilon}\right)$ for any $\varepsilon>0$ and almost all directions $\theta$.

It is plausible that the bounds like Corollary 8, Corollary 6, etc hold for any point $z \in P$, any direction $\theta \in S^{1}$, etc.

\section{Averages of Counting FunCtions}

In this section we introduce the framework of counting functions in differentiable dynamics. We will apply it to the billiard dynamics later on. Our phase spaces are "manifolds". By this we will mean compact manifolds with boundaries, corners, and singular points, in general. Our setting involves i) a foliation of the phase space by closed

\footnotetext{
${ }^{1}$ Not to be confused with the concept of universal covering space in topology.
} 
submanifolds that are fibers for a projection onto a manifold of smaller dimension; ii) a submanifold in the phase space, transversal to the fibers; iii) a weight function on the product of the phase space and the time. See the details below. The dynamics in question may be discrete or continuous. We will expose the two cases separately. The two subsections that follow are parallel, and the treatments differ in technical details.

1.1. Discrete dynamics. Let $T: X \rightarrow X, T^{-1}: X \rightarrow X$ be piecewise diffeomorphisms with the following data.

1. There is a fibration $\eta: X \rightarrow \Theta$ whose base is a compact manifold and whose fibers $R_{\theta}=\eta^{-1}(\theta)$ are compact submanifolds, such that $\operatorname{dim}\left(R_{\theta}\right)=\operatorname{dim}(X)-\operatorname{dim}(\Theta)$. We will use the notation $X=\cup_{\theta \in \Theta} R_{\theta}$. 2. There is a closed submanifold, $Y \subset X$, $\operatorname{dim}(Y)=\operatorname{dim}(\Theta)$, such that for $k \in-\mathbb{N}$ the manifolds $T^{k}(Y)$ are transversal to the fibers $R_{\theta}$.

3. There is a weight function, i. e., a continuous, non-negative function $w(x, t)$ on $X \times \mathbb{N}$. The function $w$ may depend only on time, e. g., $w=\chi_{n}$, the indicator function of $[0, n-1]$.

Remark 1. Condition 2 may be weakened, as follows.

$2^{\prime}$. There is a closed submanifold, $Y \subset X$, and a set $\Theta_{\text {ex }} \subset \Theta$ of measure zero such that for $k \in-\mathbb{N}$ and $\theta \in \Theta \backslash \Theta_{\text {ex }}$ the manifolds $T^{k}(Y)$ and $R_{\theta}$ are transversal. All of our results remain valid if we replace condition 2 by the weaker condition $2^{\prime}$. However, in our applications to polygonal billards, condition 2 may not hold only for polygons in surfaces of positive curvature. See section 3.4. To simplify the exposition, we will assume in what follows that $\Theta_{\mathrm{ex}}=\emptyset$.

In view of condition $2, \Gamma(\theta)=\left\{(x, k): x \in R_{\theta}, k \in \mathbb{N}, T^{k}(x) \in Y\right\}$ is a countable (at most) set. The sets $\Gamma_{k}(\theta)=\left\{(x, k): x \in R_{\theta}, T^{k}(x) \in\right.$ $Y\}$ are finite for all $k \in \mathbb{N}$, and $\Gamma(\theta)=\cup \Gamma_{k}(\theta)$.

We define the weighted counting function by

$$
g(\theta ; w)=\sum_{(x, k) \in \Gamma(\theta)} w(x, k)
$$

The pure counting function $g_{n}(\theta)$ corresponds to the weight $w=\chi_{n}$. We have

$$
g_{n}(\theta)=\sum_{k=0}^{n}\left|\Gamma_{k}(\theta)\right| .
$$

\footnotetext{
${ }^{2}$ By convention, $\mathbb{N}=0,1, \ldots$
} 
Proposition 1. Let $d \theta, d y$ be finite, lebesgue-class measures on $\Theta, Y$ respectively. Then for $k \in \mathbb{N}$ there are functions $r_{k}(\cdot) \geq 0$ on $Y$, determined by the data 1) and 2) alone, such that

$$
\int_{\Theta} g(\theta ; w) d \theta=\int_{Y}\left\{\sum_{k \in \mathbb{N}} w\left(T^{-k} \cdot y, k\right) r_{k}(y)\right\} d y .
$$

Proof. For any $k \in \mathbb{N}$ set $f_{k}=\eta \circ T^{-k}: Y \rightarrow \Theta$. By conditions 1 and $2, f_{k}$ is a local diffeomorphism. Therefore $f_{k}^{*}(d \theta)=r_{k}(y) d y$.

It suffices to establish equation (3) for the special case $w(x, i)=0$ if $i \neq k$. A point $x \in X$ contributes to the integral in the left hand side of equation (3) iff $T^{k} \cdot x \in Y$, or equivalently, $\eta(x)=f_{k}(y), y \in Y$. The claim follows by a straightforward change of variables.

1.2. Continuous dynamics. Let $b^{t}: \Psi \rightarrow \Psi$ be a flow of piecewise diffeomorphisms on a phase space $\Psi$ with the following data.

1. There is a fibration $q: \Psi \rightarrow Z$ with a compact base and fibers $q^{-1}(z)=R_{z} \subset \Psi$, transversal to the flow. We will use the notation $\Psi=\cup_{z \in Z} R_{z}$.

2. There is a closed submanifold, $M \subset \Psi$, $\operatorname{dim}(M)=\operatorname{dim}(Z)-1$, transversal to the flow, and such that $N=\cup_{t \in \mathbb{R}_{-}} b^{t} \cdot M$ is transversal to the fibers $R_{z} \cdot 3$

3. There is a weight function, i. e., a continuous, non-negative function $w(x, t)$ on $\Psi \times \mathbb{R}_{+}$. In a special case, $w$ depends only on time, e. g., $w=\chi_{l}$, the indicator function of $[0, l]$.

In view of condition $2, G(z)=\left\{(x, t): x \in R_{z}, 0 \leq t, b^{t}(x) \in M\right\}$ is a countable (at most) set. The sets $G_{l}(z)=\left\{(x, t): x \in R_{z}, 0 \leq t \leq\right.$ $\left.l, b^{t}(x) \in M\right\}$ are finite for all $l \in \mathbb{R}_{+}$, and $G(z)=\cup G_{l}(z)$.

We define the weighted counting function by

$$
g(z ; w)=\sum_{(x, t) \in G(z)} w(x, t) .
$$

The pure counting function $g_{l}(z)$ corresponds to the weight $w=\chi_{l}$. We have

$$
g_{l}(z)=\left|G_{l}(z)\right|
$$

Proposition 2. Let $d z, d m$ be finite, lebesgue-class measures on $Z, M$ respectively; let $d t$ be the lebesgue measure on $\mathbb{R}$. Then there exist a

\footnotetext{
${ }^{3}$ Our results remain valid if the set of parameters $Z_{\text {ex }} \subset Z$ where the transversality fails has measure zero. See Remark 1. In what follows, by condition $2^{\prime}$ we will mean the weakened condition 2 either in the setting of section 1.2 or section 1.1 .
} 
positive function $r(\cdot)$ on $M \times \mathbb{R}_{+}$, determined by the data 1) and 2), and such that

$$
\int_{Z} g(z ; w) d z=\int_{M \times \mathbb{R}_{+}} w\left(b^{-t} \cdot m, t\right) r(m, t) d m d t
$$

Proof. We define the mapping $f: M \times \mathbb{R}_{+} \rightarrow Z$ by $f=q \circ b^{-t}$. By conditions 1 and $2, f$ has full rank almost everywhere. The pullback by $f$ of $d z$ is absolutely continuous with respect to $d m d t$, hence $f^{*}(d z)=r(m, t) d m d t$.

For $0<l$ set $w_{l}(x, t)=w(x, t) \chi_{l}(t)$, and let $g_{l}(z ; w)$ be the corresponding counting function. Set $I_{l}(w)=\int_{Z} g_{l}(z ; w) d z$. A point, $x \in \Psi$, contributes to $I_{l}(w)$ iff $x \in \varphi(M \times[0, l])$. Under the change of variables $d z=d(q \circ \varphi(m, t))=r(m, t) d m d t$, we have

$$
I_{l}(w)=\int_{M \times[0, l]} w\left(b^{-t} \cdot m, t\right) r(m, t) d m d t .
$$

In the limit $l \rightarrow \infty$, we obtain the claim.

1.3. Special cases. We will discuss a few special cases of Proposition 1 and Proposition 2. First, the discrete version. The function $g_{n}(\theta)$ counts the number of visits in $Y$ of points $x \in R_{\theta}$ during the first $n$ steps of their journey. Set $\rho_{k}=\int_{Y} r_{k}(y) d y$, and $R_{n}=\sum_{k=0}^{n-1} \rho_{k}$. Then $\rho_{k}$ is the volume of $Y_{k}=T^{-k}(Y)$ with respect to the measure $\eta^{*}(d \theta)$. Proposition 1 yields

$$
\int_{\Theta} g_{n}(\theta) d \theta=R_{n}
$$

In the continuous case the function $g_{l}(z)$ counts the number of visits in $M$ of orbits $b^{t} \cdot x, x \in R_{z}$, during the period $0 \leq t \leq l$. Let $R(l)$ be the volume of the manifold $N_{l} \subset \Psi$ with respect to the measure $q^{*}(d z)$. Proposition 2 yields

$$
\int_{Z} g_{l}(z) d z=R(l)
$$

\section{Bounds ON COUNTING FUnCTIONS}

In this section we analyze the setting of section 1 from the measure theoretic viewpoint. This allows us to obtain pointwise upper bounds on counting functions in a broad spectrum of situations.

Let $X, \mu$ be a finite measure space. Let $f(x ; t)$ (for $t \in \mathbb{R}_{+}$) be a family of nonnegative $L^{1}$ functions on $X$. Set

$$
F(t)=\int_{X} f(x ; t) d \mu(x) .
$$


Lemma 1. For almost every $x \in X$ there exists $C=C(x)>0$ such that for arbitrarily large $n \in \mathbb{N}$ there is $t \geq n$ satisfying $f(x, t)<C F(t)$.

Proof. For $0<C$ and $n \in \mathbb{N}$ let

$$
B_{n}(C)=\{x \in X: C F(t)<f(x ; t) \forall t>n\},
$$

and set

$$
B(C)=\bigcup_{n \in \mathbb{N}} B_{n}(C)
$$

Integrating the inequality above, we obtain $\mu\left(B_{n}(C)\right) \leq C^{-1}$ for any $n$. Thus $\mu(B(C)) \leq C^{-1}$, and hence $\mu\left(\cap_{C \in \mathbb{R}_{+}} B(C)\right)=0$. But $\cap_{C \in \mathbb{R}_{+}} B(C) \subset$ $X$ is the complement of the set of points $x \in X$ satisfying the hypothesis of the lemma.

Let the setting be as in Lemma 1. In addition, we suppose that i) the functions $f(x ; t)$ are nondecreasing in $t$ and ii) $F(t) \rightarrow \infty$.

Lemma 2. Let $\varepsilon>0$ be arbitrary. Then for almost every $x \in X$ there exists $T=T(x, \varepsilon)>0$ such that for all $t>T$ we have

$$
f(x ; t) \leq F(t)(1+\log (1+F(t)))^{1+\varepsilon} .
$$

Proof. Denote by $f\left(x ; t^{-}\right)$(resp. $F\left(t^{-}\right)$) the limits of $f(x ; s)$ (resp. $F(s))$, as $s \rightarrow t$ from the left. For $n \in \mathbb{N}$ set $t_{n}=\inf \left\{t: F(t) \geq 2^{n}\right\}$. Then $F\left(t_{(n+1)}{ }^{-}\right) \leq 2 F\left(t_{n}\right)$. Let $A_{n} \subset X$ be the set of points satisfying the inequality

$$
f\left(x ; t_{n}^{-}\right) \leq \frac{1}{2} F\left(t_{n}^{-}\right)\left(1+\log \left(1+\frac{1}{2} F\left(t_{n}{ }^{-}\right)\right)\right)^{1+\epsilon} .
$$

It suffices to prove that the set $\bigcup_{n \in \mathbb{N}} \bigcap_{k>n} A_{k}$ has full measure. Indeed, for $x \in A_{n}$ and $t \in\left[t_{n}, t_{n+1}\right)$ we have

$$
\begin{aligned}
& f(x ; t) \leq f\left(x ; t_{n+1}^{-}\right) \leq \frac{1}{2} F\left(t_{n+1}{ }^{-}\right)\left(1+\log \left(1+\frac{1}{2} F\left(t_{n+1}{ }^{-}\right)\right)^{1+\varepsilon}\right. \\
& \leq F\left(t_{n}\right)\left(1+\log \left(1+F\left(t_{n}\right)\right)\right)^{1+\varepsilon} \leq F(t)(1+\log (1+F(t)))^{1+\varepsilon} .
\end{aligned}
$$

Thus, the points $x \in \bigcup_{n \in \mathbb{N}} \bigcap_{k>n} A_{k}$ have the property equation (10).

If $B_{n} \subset X$ is any sequence of sets, we set lim $\sup _{n \rightarrow \infty} B_{n}=\bigcap_{i \in \mathbb{N}} \bigcup_{j>i} B_{j}$. Let $B_{n}$ be the complement of $A_{n}$ in $X$. Then $\limsup _{n \rightarrow \infty} B_{n}$ is the complement of $\bigcup_{n \in \mathbb{N}} \bigcap_{k>n} A_{k}$. It remains to prove that $\mu\left(\lim \sup _{n \rightarrow \infty} B_{n}\right)=$ 0 .

By Tchebysheff inequality, we have

$$
\mu\left(B_{n}\right) \leq 2\left(1+\log \left(1+\frac{1}{2} F\left(t_{n}{ }^{-}\right)\right)\right)^{-(1+\varepsilon)} .
$$


Set $\mu_{n}=\mu\left(B_{n}\right)$. Suppose first that $F$ is a continuous function. Then $F\left(t_{n}{ }^{-}\right)=F\left(t_{n}\right)=2^{n}$. By equation (12)

$$
\mu_{n} \leq 2\left(1+\log \left(1+2^{n}\right)\right)^{-(1+\varepsilon)}
$$

hence the series $\sum \mu_{n}$ converges. Since

$$
\mu\left(\limsup _{n \rightarrow \infty} B_{n}\right) \leq \sum_{n_{0}}^{\infty} \mu_{n}
$$

for any $n_{0} \in \mathbb{N}$, the claim follows.

In general, $F$ need not be continuous. It is thus possible that $t_{n}=$ $t_{n+1}$ for some $n \in \mathbb{N}$, implying $B_{n}=B_{n+1}$. From the series $\sum \mu_{n}$ we drop the terms $\mu_{n}$ such that $B_{n}=B_{n-1}$. By equation (12), the remaining terms satisfy

$$
\mu_{n} \leq 2\left(1+\log \left(1+2^{n-2}\right)\right)^{-(1+\varepsilon)} .
$$

Now the preceding argument applies.

In sections 3, 5 we will apply these results in the billiard setting. In section 3 we will estimate the integrals equation (9), hence the bounds provided by Lemmas 1, 2 will be more specific. The propositions below anticipate these applications.

Proposition 3. Let the setting and the assumptions be as in Lemma 2. Let $0<\varepsilon$ be arbitrary.

1. Let $F(t)=O\left(t^{p}\right)$ for $0<p$. Then for almost every $x \in X$ we have $f(x ; t)=O\left(t^{p+\varepsilon}\right)$.

2. Let $F(t)=O\left(e^{a t}\right)$ for $0<a$. Then for almost every $x \in X$ we have $f(x ; t)=O\left(e^{(a+\varepsilon) t}\right)$.

Proof. The first claim is immediate from Lemma 2 and $(\log t)^{1+\varepsilon}=$ $o\left(t^{\varepsilon}\right)$. The second claim follows the same way from $t^{1+\varepsilon}=o\left(e^{\varepsilon t}\right)$.

For applications to the billiard map we need a counterpart of Proposition 3 for integer-valued time. We state it below. Its proof is analogous to the proof of Proposition 3. Moreover, the discrete time case may be formally reduced to the continuous time case. We leave details to the reader.

Let $X, \mu$ be a finite measure space. Let $f(x ; n), n \in \mathbb{N}$ be a sequence of nonnegative $L^{1}$ functions on $X$ such that for every $x \in X$ the numerical sequence $f(x ; n)$ is nondecreasing. Set $F(n)=\int_{X} f(x ; n) d \mu$.

Proposition 4. Let $0<\varepsilon$ be arbitrary. Then the following claims hold. 
1. Let $F(n)=O\left(n^{p}\right)$ for $0<p$. Then for almost every $x \in X$ we have $f(x ; n)=O\left(n^{p+\varepsilon}\right)$.

2. Let $F(n)=O\left(e^{a n}\right)$ for $0<a$. Then for almost every $x \in X$ we have $f(x ; n)=O\left(e^{(a+\varepsilon) n}\right)$.

Remark 2. All of the bounds $f(\cdot)=O(\cdot)$ in preceding propositions are equivalent to the formally stronger bounds $f(\cdot)=o(\cdot)$.

\section{Counting Functions For POlygonal Billiard}

We will now apply the preceding material to the billiard dynamics. Our billiard table will be a geodesic polygon either in the euclidean plane $\mathbb{R}^{2}$, or the round sphere $\mathbb{S}^{2}$, or the hyperbolic plane $\mathbb{H}^{2}$. We refer to [4, [7, and section 6 for the background.

\subsection{Direction counting functions for billiard maps in euclidean} polygons. Let $P \subset \mathbb{R}^{2}$ be a euclidean polygon, and let $T: X(P) \rightarrow$ $X(P)$ be the billiard map. Elements of the phase space $X=X(P)$ are oriented geodesic segments in $\mathbb{R}^{2}$ with endpoints in $\partial P$. A segment $x \in X$ ending in a corner of $P$ is singular; the element $T x$ is not well defined. A billiard orbit $x, T x, \ldots, T^{k-1} x$ is a singular orbit of length $k$ if $T^{k-1} x$ is the first singular element in the sequence.

Assigning to $x \in X$ its direction, $\eta(x) \in S^{1}$, we obtain a fibration $\eta: X \rightarrow S^{1}$ with fibers $R_{\theta} \subset X$. See figure 1. We define the counting function $g d_{\theta}(n)$ for singular orbits in direction $\theta$ as the number of phase points $x \in R_{\theta}$ that yield singular orbits of length $k \leq n$.

Theorem 1. Let $P \subset \mathbb{R}^{2}$ be an arbitrary polygon. Let $K(P)$ be the set of its corners. Let $\alpha(v)$ be the angle of $v \in K(P)$. Let $d \theta$ be the lebesgue measure on $S^{1}$.

Let $K \subset K(P)$. Then

$$
\int_{S^{1}} \sum_{v \in K} g d_{\theta}(n ; v) d \theta=\left(\sum_{v \in K} \alpha(v)\right) n .
$$

Proof. It suffices to prove the claim for a singleton, $K=\{v\}$. Let $Y=Y(v) \subset X$ be the set of segments $x \in X$ ending at $v$. Let $d y$ be the angular measure on $Y$. These data fit into the setting of section 1.1, and $g d_{\theta}(n ; v)$ is the pure counting function.

Let $B(z, \alpha)$ be a conical beam of light with apex angle $\alpha$ emanating from $z \in \mathbb{R}^{2}$. After reflecting in $\partial P$, it splits into a finite number of beams $B\left(z_{i}, \alpha_{i}\right)$ satisfying $\sum \alpha_{i}=\alpha$. The preservation of light volume is due to the flatness of $\partial P$. 
By preceding remark, the functions $r_{k}(\cdot)$ of Proposition 1 satisfy $r_{k}(\cdot) \equiv 1$. The claim now follows from the special case of Proposition 1 considered in section 1.3 .

Let $p, q$ be the numbers of corners, obstacles in $P$. Let $\kappa(P)=$ $p+2 q-2$. Thus, $P$ is simply connected iff $q=0$ iff $\kappa(P)=p-2$.

Corollary 1. Let $P \subset \mathbb{R}^{2}$ be an arbitrary polygon. Then

$$
\int_{S^{1}} g d_{\theta}(n) d \theta=\pi \kappa(P) n
$$

Proof. Follows from Theorem 1 via $\sum_{v \in K(P)} \alpha(v)=(p+2 q-2) \pi$.

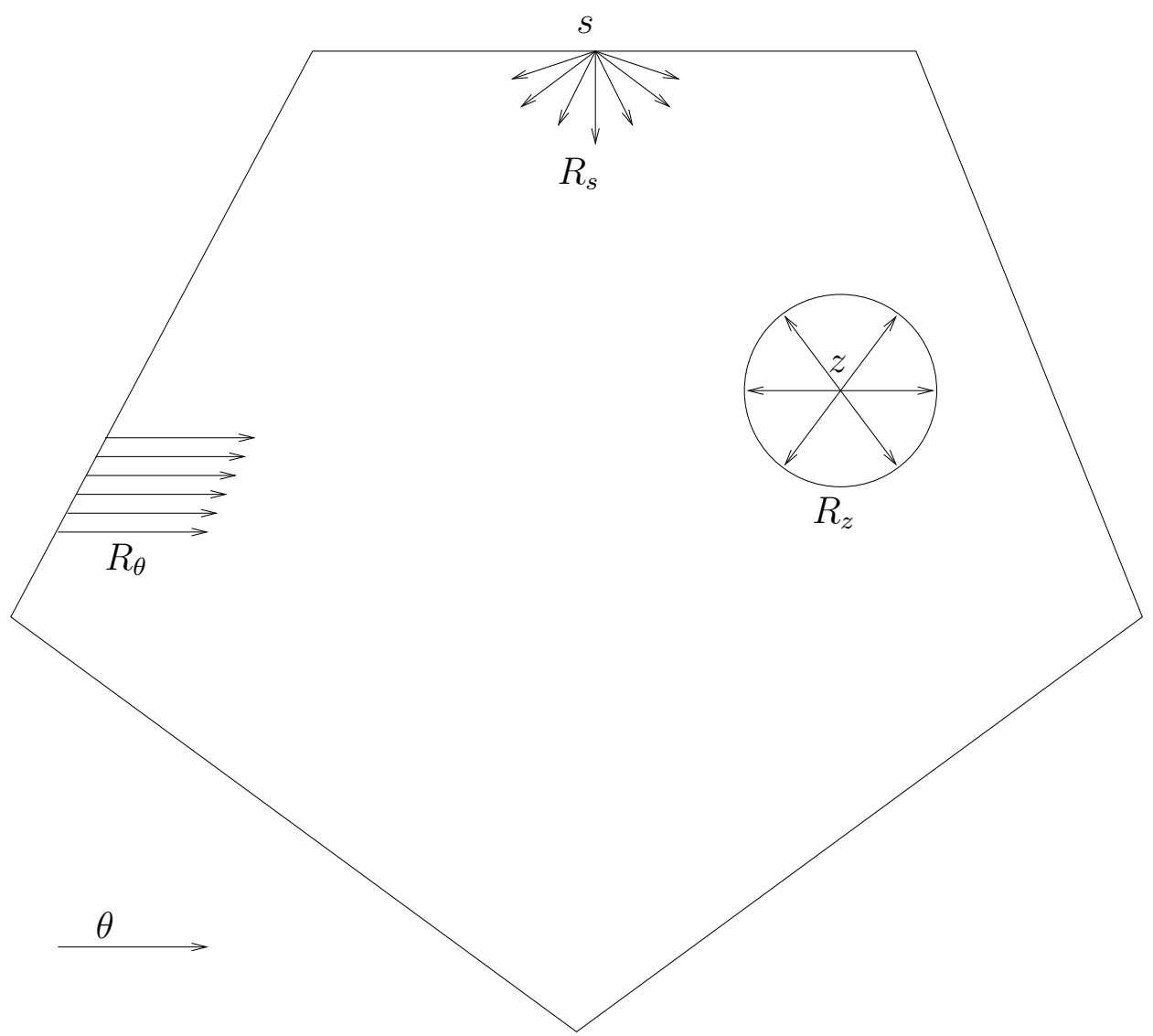

Figure 1. Base sets for billiard counting functions 


\subsection{Position counting functions for billiard flows in euclidean} polygons. Let $P \subset \mathbb{R}^{2}$ be a polygon, and let $b^{t}: \Psi \rightarrow \Psi$ be the billiard flow. See section 6 for details. For $z \in P$ and $v \in K(P)$ let $g c_{z}(l ; v)$ be the number of billiard flow orbits that start from $z \in P$ and wind up at $v$ by time $l$. Then $g c_{z}(l)=\sum_{v \in K(P)} g c_{z}(l ; v)$ is the number of singular billiard orbits of length at most $l$ starting from $z$. This is the position counting function for the billiard flow in $P$.

Theorem 2. Let $P \subset \mathbb{R}^{2}$ be a euclidean polygon, and let $d z$ be the lebesgue measure on $P$. Then for any $K \subset K(P)$ we have

$$
2 \int_{P} \sum_{v \in K} g c_{z}(l ; v) d z=\left(\sum_{v \in K} \alpha(v)\right) l^{2} .
$$

Proof. It suffices to prove the claim for $K=\{v\}$. We view elements of $\Psi$ as pairs $z, \theta$ where $z \in P$ is the basepoint, and $\theta$ is the direction. Let $M=\{(v, \theta):(v,-\theta) \in \Psi\}$. Let $q: \Psi \rightarrow P$ be the obvious projection. Its fibers $R_{z}$ are the base sets for the counting functions $g c_{z}(l ; v)$. See figure 1. Set $w=\chi_{l}$. These data satisfy the assumptions of Proposition 2, and $g c_{z}(l ; v)$ is the pure counting function.

We set $d m$ to be the angular measure, and compute the function $r(m, t)$ in equation (6). By Corollary 13 in section 6, $r=t \chi_{l}$. Proposition 2 implies the claim.

When $K=K(P)$, the left hand side in equation (15) is the average of the position counting function. The argument of Corollary 1 yields the following.

Corollary 2. Let $P \subset \mathbb{R}^{2}$ be an arbitrary polygon. Then

$$
2 \int_{P} g c_{z}(l) d z=\pi \kappa(P) l^{2}
$$

\subsection{Position counting functions for billiard maps in euclidean}

polygons. We will now discuss two billiard map analogs of the preceding example. Let $P \subset \mathbb{R}^{2}$ be a euclidean polygon, and let $T$ : $X(P) \rightarrow X(P)$ be the billiard map. The phase space $X=X(P)$ consists of pairs $(s, \alpha)$ where $s$ is the arclentgh parameter on $\partial P$, and $0<\alpha<\pi$ is the outgoing angle. See [4, 7] and section 6] for details. An orbit $x, T x, \ldots, T^{k-1} x$ is singular, of (combinatorial) length $k$ if its last segment ends at a corner of $P$.

Let $s \in \partial P, v \in K(P)$. Define $G D_{s}(n ; v)$ to be the set of phase points $(s, \alpha) \in X$ whose orbits of length less than or equal to $n$ end at 
$v$. Set

$$
g d_{s}(n ; v)=\left|G D_{s}(n ; v)\right|, \operatorname{god}_{s}(n ; v)=\sum_{(s, \alpha) \in G D_{s}(n ; v)} \sin \alpha .
$$

The expressions

$$
g d_{s}(n)=\sum_{v \in K(P)} g d_{s}(n ; v), \operatorname{god}_{s}(n)=\sum_{v \in K(P)} \operatorname{god}_{s}(n ; v)
$$

are the pure position counting function and the optical position counting function for the billiard map in $P$.

Let $z \in \mathbb{R}^{2}$ and let $\gamma \subset \mathbb{R}^{2}$ be an oriented piecewise $C^{1}$ curve. Denote by $d_{z} s$ the projection of the arclength form $d s$ of $\gamma$ onto the direction perpendicular to the line from $z$ to $s \in \gamma$. The integral $\int_{\gamma} d_{z} s=|\operatorname{opt}(\gamma, z)| \leq|\gamma|$ is the optical length of $\gamma$ viewed from $z$.

Let $z \in P$. Unfolding $k$-segment billiard orbits emanating from $z$, we obtain a set of linear segments in $\mathbb{R}^{2}$. Let $\partial_{z}(P ; k) \subset \mathbb{R}^{2}$ be the curve traced by their endpoints. We say that $\partial_{z}(P ; k) \subset \mathbb{R}^{2}$ is the outer boundary of $P$, as viewed from $z$, after $k$ iterates.

Theorem 3. Let $P$ be a euclidean polygon, and let $K \subset K(P)$ be a set of corners. Then

$$
\begin{gathered}
\int_{\partial P} \sum_{v \in K} g d_{s}(n ; v) d s=\sum_{v \in K} \sum_{k=1}^{n}\left|\partial_{v}(P ; k)\right| \\
\int_{\partial P} \sum_{v \in K} \operatorname{god}_{s}(n ; v) d s=\sum_{v \in K} \sum_{k=1}^{n}\left|\operatorname{opt}\left(\partial_{v}(P, k)\right)\right| .
\end{gathered}
$$

Proof. It suffices to prove the claims for a singleton, $K=\{v\}$. Let $\eta$ : $X \rightarrow \partial P$ be the natural projection. Using the arclength parametrization, we identify $\partial P$ with the interval $[0,|\partial P|] \subset \mathbb{R}$. For $0 \leq s \leq|\partial P|$ let $R_{s}=\eta^{-1}(s) \subset X$ be the fiber. Then $R_{s}$ are the base sets for the counting functions $g d_{s}(n ; v), \operatorname{god}_{s}(n ; v)$. See figure 11. Let $Y=$ $Y(v) \subset X$ be the set of phase points whose $T^{-1}$-orbits emanate from $v$. The assumptions of section [1.1 are satisfied. The weight functions are $w(s, \alpha, t)=\chi_{n}(t)$ and $w_{o}(s, \alpha, t)=\sin \alpha \cdot \chi_{n}(t)$ for the two cases at hand. Let $\varphi$ be the angle parameter on $Y$. The measures on $\partial P$ and $Y$ have densities $d s$ and $d \varphi$ respectively.

The integrals in the right hand side of equation (3) are over the curves $\partial_{v}(P ; k), 0 \leq k \leq n-1$. The integrands are $d s(\varphi)$ and $\sin \alpha \cdot d s(\varphi)=$ $d_{v} s(\varphi)$ in respective cases.

We will need estimates on lengths and optical lengths. 
Lemma 3. For any polygon $P \subset \mathbb{R}^{2}$ there exist $0<c_{1}<c_{2}<\infty$ such that for $n$ sufficiently large

$$
c_{1} n^{2} \leq \sum_{v \in K} \sum_{k=1}^{n}\left|\operatorname{opt}\left(\partial_{v}(P, k)\right)\right| \leq c_{2} n^{2}, \quad c_{1} n^{2} \leq \sum_{v \in K} \sum_{k=1}^{n}\left|\left(\partial_{v}(P, k)\right)\right| .
$$

Proof. There exist positive constants $d_{1}, d_{2}$ and $m_{0} \in \mathbb{N}$, such that for any orbit $\gamma$ of the billiard map with $m>m_{0}$ segments, we have $d_{1}|\gamma| \leq m \leq d_{2}|\gamma|$ [4.

Let $v \in K(P)$. We will estimate $\sum_{k=m_{0}}^{n}\left|\operatorname{opt}\left(\partial_{v}(P, k)\right)\right|$, as $n \rightarrow \infty$. Let $\theta_{1} \leq \theta \leq \theta_{2}$ be the angular parameter for orbits emanating from $v$; let $r(\theta)$ be the geometric length of the orbit. Suppose that $r_{1} \leq r(\theta) \leq$ $r_{2}$. Then the optical length in question is sandwiched between the lengths of circular arcs of radii $r_{1}, r_{2}$ of angular size $\theta_{2}-\theta_{1}$. By preceding remarks, if $k$ is sufficiently large, the bounds $r_{1}, r_{2}$ are proportional to $k$. The total angular size does not depend on $k$. Hence, for sufficiently large $k$ we have linear upper and lower bounds on $\sum_{v \in K}\left|\operatorname{opt}\left(\partial_{v}(P, k)\right)\right|$. The other inequality follows from $\left|\operatorname{opt}\left(\partial_{v}(P, k)\right)\right| \leq\left|\partial_{v}(P, k)\right|$.

\subsection{Position counting functions for billiard flows in spherical} polygons. The study is analogous to the planar case discussed in section [3.2, we will use the same notation whenever this does not lead to confusion. We denote by $d z$ the lebesgue measure on $\mathbb{S}^{2}$, and by $\alpha(v)$ the angle of a corner of $P$. Set

$$
\zeta(x)=1-\cos x-\frac{2}{\pi} x .
$$

Theorem 4. Let $P \subset \mathbb{S}^{2}$ be a geodesic polygon, and let $K \subset K(P)$. Then

$$
\int_{P} \sum_{v \in K} g c_{z}(l ; v) d z=\left(\sum_{v \in K} \alpha(v)\right)\left(\frac{2}{\pi} l+\zeta(l-\pi\lfloor l / \pi\rfloor)\right) .
$$

Proof. It suffices to prove the claim when $K=\{v\}$. Let $M=M(v) \subset$ $\Psi$ be as in section 3.2 , and let $d \alpha$ be the angular measure on it. The assumptions 1, 3 of section 1 are satisfied; the transversality of $b^{t} \cdot M$ and $R_{z}$ may fail for at most a countable set of parameters $P_{\mathrm{ex}} \subset P$. See Remark 3 in section 6. Hence, condition $2^{\prime}$ is fullfilled, and the results of section 1.2 hold. The function $g c_{z}(l ; v)$ is a pure counting function. The claim now follows from Proposition 2 and Corollary 13 ,

Let $\kappa(P)$ be as in section 3.1 . 
Corollary 3. Let $P \subset \mathbb{S}^{2}$ be an arbitrary polygon. Then

$$
\int_{P} g c_{z}(l) d z=(\kappa(P) \pi+\operatorname{area}(P))\left(\frac{2}{\pi} l+\zeta(l-\pi\lfloor l / \pi\rfloor)\right) .
$$

Proof. For a spherical polygon we have $\sum_{v \in K(P)} \alpha(v)=\operatorname{area}(P)+$ $\kappa(P) \pi$. Substitute this into equation (21).

\subsection{Position counting functions for billiard flows in hyper-} bolic polygons. Our treatment and our notation are modelled on section 3.4. We denote by $d z$ the lebesgue measure on $\mathbb{H}^{2}$, and by $\alpha(v)$ the angles of corners.

Theorem 5. Let $P \subset \mathbb{H}^{2}$ be a geodesic polygon, and let $K \subset K(P)$. Then

$$
\int_{P} \sum_{v \in K} g c_{z}(l ; v) d z=\left(\sum_{v \in K} \alpha(v)\right) \cosh l .
$$

Proof. We repeat verbatim the proof of Theorem 4, and use claim 2 in Corollary 13.

Let $\kappa(P)$ be as in section 3.1

Corollary 4. Let $P \subset \mathbb{H}^{2}$ be a polygon. Then

$$
\int_{P} g c_{z}(l) d z=(\kappa(P) \pi-\operatorname{area}(P)) \cosh l .
$$

Proof. Repeat the argument of Corollary 3 , use the formula $\sum_{v \in K(P)} \alpha(v)=$ $\kappa(P) \pi-\operatorname{area}(P)$ relating the angles and the area of geodesic polygons in $\mathbb{H}^{2}$.

\section{Relating partial Complexities and COUNTing FUnCtions}

In this section we establish a framework that will allow us to study the complexity of a wide class of dynamical systems. Our motivation comes from the billiard dynamics. In fact, polygonal billiard is the target of applications for our results. The framework is more general, however. The following observations served as our guiding principles. First, natural partitions of the billiard-type systems are geared to the singularities. Second, the billiard dynamics satisfies a certain convexity property that is instrumental in the study of complexity. These principles are manifest in the framework of piecewise convex transformations [7.

There are two approaches to the billiard dynamics: The billiard flow and the billiard map. See section 6. The framework of piecewise convex 
transformations is geared to the billiard map. We begin by establishing its counterpart for flows.

4.1. Piecewise convex transformations and piecewise convex flows. A piecewise convex transformation is a triple $(X, \Gamma, T)$, where $X$ is a two-dimensional convex cell complex, $\Gamma \subset X$ is the graph formed by the union of one-cells, and $T: X \rightarrow X$ is an invertible map, regular on the two-cells of the complex, and compatible with the convex structure [7].

Let $\Psi$ be a compact manifold, with boundary and corners, in general. Let $b^{t}: \Psi \rightarrow \Psi$ be a flow, possibly with singularities; let $X \subset \Psi$ be a cross-section. We will assume that the singular set of the flow is contained in $X$. For $z \in X$ let $\tau_{+}(z), \tau_{-}(z)$ be the times when $z \in \Psi$ first reaches $X$ under $b^{t}, b^{-t}$ for $0<t$. We assume that for any $z \in \Psi \backslash X$ there is $0<\varepsilon=\varepsilon(z)$ such that $b^{t}(z)$ is regular for $|t|<\varepsilon$.

A piecewise convex flow is determined by the following data: A flow, $b^{t}: \Psi \rightarrow \Psi$, a cross-section, $X \subset \Psi$, and the structure of a convex cell complex on $X$, compatible with the poincare map. Billiard flows for polygons on surfaces of constant curvature are piecewise convex flows [7.

4.2. Partial complexities for maps and flows. Let $\left(X, \Gamma_{n}, T^{n}\right)$ be the iterates of a piecewise convex transformation $(X, \Gamma, T)$ Let $F\left(\Gamma_{n}\right)$ be the finite set of open faces of $\Gamma_{n}$; these are the continuity regions for $T^{n}$. The function $f(n)=\left|F\left(\Gamma_{n}\right)\right|$ is the (full) complexity of $(X, \Gamma, T)$.

Let $R \subset X$ be a closed subset. Set

$$
F_{R}(n)=\left\{A \in F\left(\Gamma_{n}\right): A \cap R \neq \emptyset\right\} .
$$

Definition 1. The function $f_{R}(n)=\left|F_{R}(n)\right|$ is the partial complexity of the piecewise convex transformation $(X, \Gamma, T)$ based on the subset $R$.

Let $b^{t}: \Psi \rightarrow \Psi$ be a piecewise convex flow, and let $R \subset \Psi$ be a closed, convex set transversal to the flow. For $0<l$ let $O_{R}(l)$ be the set of regular flow orbits of length $l$ starting from $R$. Let $\alpha_{0}, \alpha_{1} \in O_{R}(l)$. A homotopy is a continuous family of regular orbits $\alpha_{p} \in O_{R}(l), 0 \leq p \leq$ 1 , interpolating between $\alpha_{0}, \alpha_{1}$. We will say, for brevity, that the orbits $\alpha_{0}, \alpha_{1}$ are $R$-homotopic. We denote by $H_{R}(l)$ the set of $R$-homotopy classes.

Definition 2. The function $h_{R}(l)=\left|H_{R}(l)\right|$ is the partial complexity (based on $R$ ) of the piecewise convex flow $b^{t}: \Psi \rightarrow \Psi$.

\footnotetext{
4 They are piecewise convex transformations as well [7].
} 

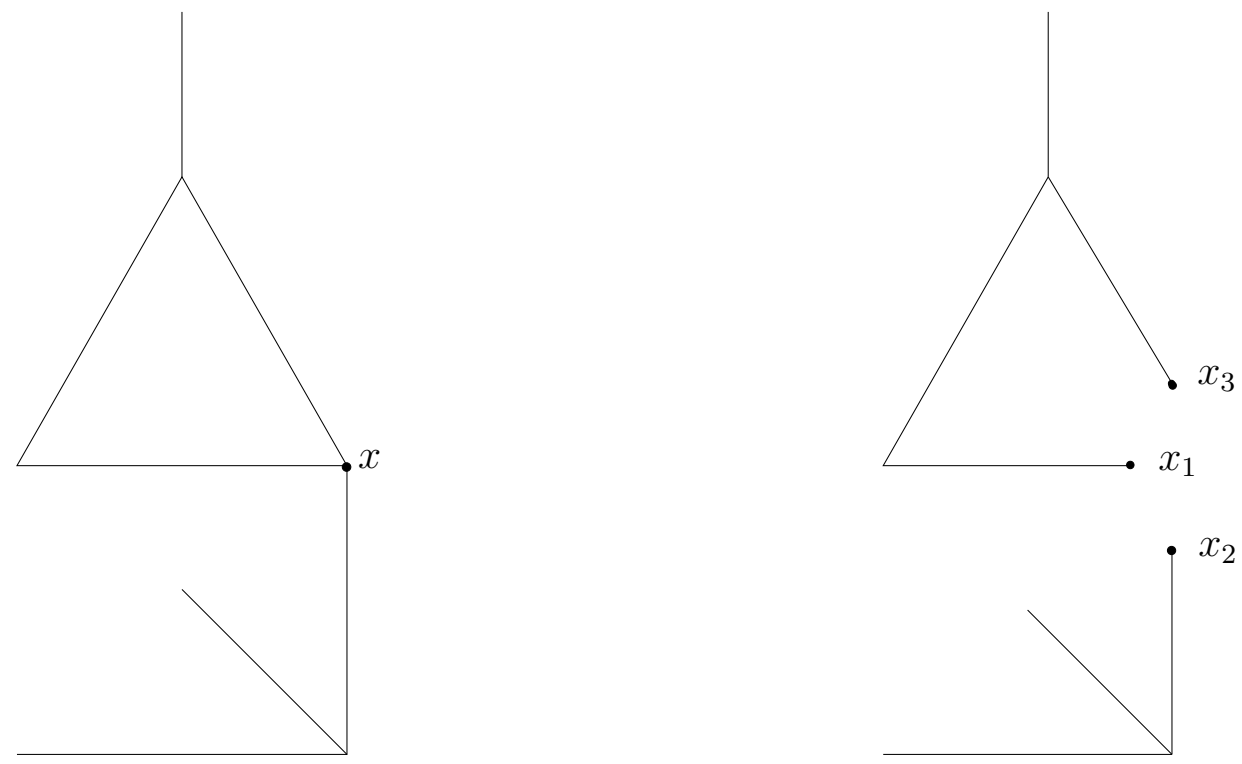

FiguRE 2. Removing a vertex in a graph

4.3. Partial complexities and counting functions. In what follows we assume that $R \subset \Psi$ is a convex graph without isolated vertices. For $x \in R$ its valence $\operatorname{val}(x)$ is the number of edges of $x$ minus one. In particular, if $x$ is an interior point of an edge, then $\operatorname{val}(x)=1$. Set $\operatorname{val}(R)=\max _{x \in R} \operatorname{val}(x)$. We endow $R \backslash\{x\}$ with the graph structure where $x$ is replaced by $1+\operatorname{val}(x)$ vertices; each of them is the endpoint of a unique edge. If $x, y, z, \ldots \in R$ are distinct points, then the inductively defined graph structure on $R$ without $x, y, z, \ldots$ does not depend on the order of removing these points. We will denote this graph by $R \backslash\{x, y, z, \ldots\}$. See figure 2 for an illustration.

Let $E(R)$ and $V(R)$ be the sets of edges and vertices, and let $c(R)$ be the number of connected components of the graph. Let $h_{i}=h_{i}(R)$ be the betti numbers of $R$, and set $\chi(R)=|V(R)|-|E(R)|$. Then $c(R)=h_{0}, \chi(R)=h_{0}-h_{1}$.

Lemma 4. Let $R$ be a finite graph, and let $x_{1}, \ldots, x_{p} \in R$ be distinct points. Then

$$
\chi(R)+\sum_{i=1}^{p} \operatorname{val}\left(x_{i}\right) \leq c\left(R \backslash\left\{x_{1}, \ldots, x_{p}\right\}\right) \leq c(R)+\sum_{i=1}^{p} \operatorname{val}\left(x_{i}\right) .
$$

If $R$ is a forest, then the bound on the right in equation (25) becomes an equality. 
Proof. It suffices to prove the claims when $R$ is connected, and we remove a single vertex, $x$. Equation (25) becomes

$$
\chi(R)+\operatorname{val}(x) \leq c(R \backslash\{x\}) \leq c(R)+\operatorname{val}(x) .
$$

We have $|V(R \backslash\{x\})|=|V(R)|+\operatorname{val}(x),|E(R \backslash\{x\})|=|E(R)|$, and $\chi(R \backslash\{x\})=\chi(R)+\operatorname{val}(x)$. Equivalently, we have $\chi(R \backslash\{x\})=h_{0}(R)+$ $\operatorname{val}(x)-h_{1}(R)$ and $h_{0}(R \backslash\{x\})=h_{0}(R)+\operatorname{val}(x)+\left(h_{1}(R \backslash\{x\})-h_{1}(R)\right)$. The former (resp. latter) identity implies the left (resp. right) inequality in equation (26).

When $R$ is a tree, we have $c(R \backslash\{x\})=c(R)+\operatorname{val}(x)$, and the remaining claim follows.

We will introduce counting functions for singular orbits of the billiard map and the billiard flow.

By definition, an orbit $\alpha=\left\{b^{t}(z), 0 \leq t \leq l\right\}$, does not pass through singular points in $\Psi$. It is regular if it does not contain any singular points in $\Psi$; it is singular if one of its endpoints is singular. The set $S_{R}(l)$ of singular orbits of length at most $l$, based in $R$, is finite. The quantities $g c_{R}(l)=\left|S_{R}(l)\right|$ and $g d_{R}(n)=\left|R \cap \Gamma_{n}\right|$ are the counting functions for singular orbits based in $R$ for the flow and the map respectively.

Now we will relate partial complexities and counting functions. We do this for a piecewise convex flow $b^{t}: \Psi \rightarrow \Psi$ and for a piecewise convex transformation $(X, \Gamma, T)$. In both cases the partial complexity is based on a 1-dimensional subset, say $R$. Recall that $g c_{R}(l), g d_{R}(n)$ are the respective counting functions, and $h_{R}(l), f_{R}(n)$ are the respective complexities. We will refer to these situations as the continuous case and the discrete case respectively.

Proposition 5. Let the setting be as above. Then the following statements hold.

1. In the continuous case there exist $h_{0} \in \mathbb{N}$ and $l_{0} \in \mathbb{R}_{+}$such that $h_{R}(l)=h_{0}+g c_{R}(l)$ for $l_{0} \leq l$. 2. In the discrete case there exist $f_{0}, n_{0} \in \mathbb{N}$ such that for $n_{0} \leq n$ we have $f_{R}(n)=f_{0}+g d_{R}(n)$.

Proof. In both cases the graph $R$ is equipped with a tower of finite sets, say $X(l)$ and $X_{n}$ respectively. Let $X_{\infty} \subset R$ be their union. We will compare the number of connected components of graphs $R \backslash X(l), R \backslash X_{n}$ with the cardinalities of these sets.

We consider the discrete case, leaving the continuous case to the reader. Let $m<n$ be any pair of natural numbers. By (the proof of) 
Lemma 4 .

$c\left(R \backslash X_{n}\right)-c\left(R \backslash X_{m}\right)=\left[h_{1}\left(R \backslash X_{n}\right)-h_{1}\left(R \backslash X_{m}\right)\right]+\sum_{x \in X_{n} \backslash X_{m}} \operatorname{val}(x)$.

We have $h_{1}\left(R \backslash X_{n}\right) \leq h_{1}\left(R \backslash X_{m}\right)$; the inequality holds iff $X_{n} \backslash$ $X_{m}$ breaks cycles in $R \backslash X_{m}$. Since the sequence $h_{1}\left(R \backslash X_{k}\right) \in \mathbb{N}$ is nonincreasing, it stabilizes. Thus, there exists $n_{1} \in \mathbb{N}$ such that for $n_{1} \leq m<n$ we have $h_{1}\left(R \backslash X_{n}\right)=h_{1}\left(R \backslash X_{m}\right)$.

The set of points $x \in R$ satisfying $1<\operatorname{val}(x)$ is finite. Thus, there exists $n_{2} \in \mathbb{N}$ such that if $n_{2} \leq k$ and $x \in X_{\infty} \backslash X_{k}$, then $\operatorname{val}(x)=1$.

Set $n_{0}=\max \left(n_{1}, n_{2}\right)$. Then for $n_{0} \leq m<n$ the above equation yields $c\left(R \backslash X_{n}\right)-c\left(R \backslash X_{m}\right)=\left|X_{n} \backslash X_{m}\right|$. Specializing to $m=n_{0}$, we obtain $f_{R}(n)=\left(f_{R}\left(n_{0}\right)-g d_{R}\left(n_{0}\right)\right)+g d_{R}(n)$.

\section{BOUNDS ON PARTIAL COMPLEXITIES FOR THE BILLIARD}

We will use the preceding material to derive bounds on partial complexities for the polygonal billiard.

5.1. Direction complexities for billiard maps in euclidean polygons. We use the setting and the notation of section 3.1. For a polygon $P$ and a direction $\theta$, we denote by $f d_{\theta}(n)$ the partial complexity with base $R_{\theta}$. This is the complexity in direction $\theta$.

Corollary 5. For lebesgue almost all directions $\theta$ there is $C=C(\theta)$ and there are arbitrarily large $n$ such that $f d_{\theta}(n) \leq C n$.

Proof. Each $R_{\theta}$ is a convex graph in the phase space [7]. By Lemma 1 and Corollary 1 , the counting functions $g d_{\theta}(n)$ have the desired properties. By the second claim of Proposition 5, the directional complexities do as well.

Corollary 6. For any $\varepsilon>0$ and almost every direction $\theta$ we have $f d_{\theta}(n)=O\left(n^{1+\varepsilon}\right)$.

Proof. The proof goes along the lines of the proof of Corollary 5, Instead of Lemma 1, we use Proposition 4 (the first claim).

5.2. Position complexities for billiard flows in euclidean polygons. Let $P$ be a euclidean polygon, and let $z \in P$ be any point. We consider the billiard flow in $P$, and use the setting of section 3.2 . Thus, $g c_{z}(l)$ is the position counting function for orbits emanating from $z$. We denote by $h_{z}(l)$ the corresponding partial complexity.

Corollary 7. For almost every point $z$ there is a positive number $C=$ $C(z)$ such that $h_{z}(l) \leq C l^{2}$ for arbitrarily large $l$. 
Proof. The sets $R_{z}$ satisfy the assumptions of section 4. The claim follows from Lemma 1, Corollary 2 and the continuous case in Proposition 5 .

Corollary 8. For any $\varepsilon>0$ and almost every $z \in P$ we have $h_{z}(l)=$ $O\left(l^{2+\varepsilon}\right)$.

Proof. The proof is similar to the preceding argument, and we use the first claim in Proposition 3 instead of Lemma 1 .

5.3. Position complexities for billiard maps in euclidean polygons. This is the billiard map analog of the preceding example. Let $P$ be a euclidean polygon, and let $s \in \partial P$. We use the setting of section 3.3. There we have defined the counting functions $g d_{s}(n), \operatorname{god}_{s}(n)$. Let $f_{s}(n)$ be the partial complexity corresponding to $g d_{s}(n)$. This is the position complexity for the billiard map.

Corollary 9. Let $P \subset \mathbb{R}^{2}$ be a polygon such that $\sum_{v \in K} \sum_{k=1}^{n}\left|\partial_{v}(P ; k)\right|$ has a quadratic upper bound 5 Then for almost all $s \in \partial P$ we have $f_{s}(n)=O\left(n^{2+\varepsilon}\right)$ for any $0<\varepsilon$.

Proof. The sets $R_{s} \subset X$ satisfy the assumptions of section 4. We use Theorem 3, Lemma 3, and apply Proposition 5.

The estimate of Corollary 9 on $f_{s}(n)$ is conditional, because in general we have no efficient upper bound on $\sum_{k=1}^{n}\left|\partial_{v}(P ; k)\right|$.

\subsection{Position complexities for billiard flows in spherical poly-} gons. We use the setting of section [3.4. For a spherical polygon, $P \subset S^{2}$, and $z \in P$, let $h_{z}(l)$ be the position complexity.

Corollary 10. For almost every point $z \in P$ there is $C=C(z)$ and there are arbitrarily large $l$ such that $h_{z}(l) \leq C l$.

Proof. The sets $R_{z}$ satisfy the assumptions of section 4. We use Lemma1, Corollary 3 , and Proposition 5 .

Corollary 11. For any $\varepsilon>0$ and almost every $z \in P$ we have $h_{z}(l)=$ $O\left(l^{1+\varepsilon}\right)$.

Proof. See the proof of Corollary 8.

\footnotetext{
${ }^{5}$ This holds if $P$ is a rational polygon [11].
} 
5.5. Position complexities for billiard flows in hyperbolic polygons. This material is the hyperbolic plane counterpart of section 3.2 , and we use the setting of section 3.5.

Corollary 12. Let $P \subset \mathbb{H}^{2}$ be a geodesic polygon, let $z \in P$, and let $h_{z}(l)$ be the position complexity. Then for almost every point $z \in P$ we have $h_{z}(l)=O\left(e^{(1+\varepsilon) l}\right)$.

Proof. We verify that the sets $R_{z}$ satisfy the assumptions of section 4 , and mimick the proof of Corollary 8, we use Corollary 4, Proposition 3, and the continuous case of Proposition 5 .

\section{Appendix: Covering spaces for polygonal Billiards}

Let $M$ be a simply connected surface of constant curvature, and let $P \subset M$ be a connected geodesic polygon. We normalize the metric so that the curvature is either zero $\left(M=\mathbb{R}^{2}\right)$, or one $\left(M=\mathbb{S}^{2}\right)$, or minus one $\left(M=\mathbb{H}^{2}\right)$.

Let $A$ be the set of sides in $P$. We will denote its elements by $a, b, \ldots$ For a side, say $a \in A$, let $s_{a} \in \operatorname{Iso}(M)$ be the corresponding geodesic reflection. We associate with $P$ a Coxeter system $(G, A)[2]$. We denote by $\sigma_{a}, \sigma_{b}, \cdots \in G$ the elements corresponding to $a, b, \ldots \in A$. They generate $G$. The defining relations are $\sigma_{a}^{2}=1$ and $\left(\sigma_{a} \sigma_{b}\right)^{n(a, b)}=1$; the latter arise only for the sides $a, b$ with a common corner if the angle, $\theta(a, b)$, between them is $\pi$-rational. In this case $n(a, b)$ is the denominator of $\theta(a, b) / \pi$. Otherwise $n(a, b)=\infty$.

To any "generalized polyhedron" $P$ corresponds a topological space $\mathfrak{C}$ endowed with several structures, and a Coxeter system [2]. Our situation fits into the framework of [2], and we apply its results. First, $\mathfrak{C}$ is a differentiable surface. Second, $\mathfrak{C}$ is tiled by subsets $P_{g}, g \in G$, labelled by elements of the Coxeter group $G$; we call them the tiles, and identify $P_{e}$ with $P$. The group $G$ acts on $\mathfrak{C}$ properly discontinuously, preserving the tiling: $g \cdot P_{h}=P_{g h}$.

Since $P_{e}$ is identified with $P \subset M$, it inherits from $M$ a riemannian structure. The action of $G$ is compatible with this structure, and extends it to all of $\mathfrak{C}$. This riemannian structure generally has cone singularities at vertices of the tiling $\mathfrak{C}=\cup_{g \in G} P_{g} 6$ Around other points this riemannian structure is isometric to that of $M$; in particular, except for cone points, $\mathfrak{C}$ has constant curvature. The group $G$ acts on $\mathfrak{C}$ by isometries.

\footnotetext{
${ }^{6}$ Each vertex, $v$, corresponds to a corner of $P$. The metric at $v$ is regular iff the corner angle is $\pi / n, n=2,3, \ldots$.
} 
Definition 3. The space $\mathfrak{C}$ endowed with the riemannian structure, the isometric action of $G$ and the $G$-invariant tiling $\mathfrak{C}=\cup_{g \in G} P_{g}$ is the universal covering space of the geodesic polygon $P \subset M$.

If $X$ is a riemannian manifold (with boundary and singularities, in general), we denote by $T X=\cup_{x \in X} T_{x} X$ its unit tangent bundle. The classical construct of geodesic flow, $G_{X}^{t}: T X \rightarrow T X$, extends to manifolds with boundaries and singularities. In particular, $G_{X}^{t}$ makes sense when $X=M, P$, or $\mathfrak{C}$. Another classical construct, the exponential map, also extends to our situation. For $x \in X$ as above, and $(v, t) \in T_{x} X \times \mathbb{R}_{+}$, we set $\exp _{X}(v, t) \in X$ be the base-point of $G_{X}^{t}(v)$. We will use the notation $\exp _{X}^{x}$ to indicate that we are exponentiating from the point $x$. If $X$ is nonsingular, then $\exp _{X}^{x}: T_{x} X \times \mathbb{R}_{+} \rightarrow X$ is a differentiable mapping. For $X$ with singularities, such as our $P$ and $\mathfrak{C}$, the maps $\exp _{X}^{x}$ are defined on proper subsets of $T_{x} X \times \mathbb{R}_{+}$; these subsets have full lebesgue measure. Generally, the maps do not extend by continuity to all of $T_{x} X \times \mathbb{R}_{+}$.

Let $X, Y$ be nonsingular riemannian manifolds of the same dimension; let $\varphi: X \rightarrow Y$ be a local isometry. It induces a local diffeomorphism $\Phi: T X \rightarrow T Y$ commuting with the geodesic flows: $\Phi \circ G_{X}^{t}=G_{Y}^{t} \circ \Phi$. The exponential maps commute as well: $\varphi \circ \exp _{X}^{x}=$ $\exp _{Y}^{\varphi(x)} \circ d_{x} \varphi$. These relationships hold, in particular, for coverings of nonsingular riemannian manifolds. Suitably interpreted, they extend to (branched) coverings of riemannian manifolds with boundaries, corners, and singularities. In our case $X=\mathfrak{C}$, while $Y=M$, or $Y=P$. We will now define the mappings $f: \mathfrak{C} \rightarrow P, F: T \mathfrak{C} \rightarrow T P$ and $\varphi: \mathfrak{C} \rightarrow M, \Phi: T \mathfrak{C} \rightarrow T M$.

The identification $P_{e}=P$ defines $f, \varphi$ on $P_{e}$. To extend them to all of $\mathfrak{C}$, we use the tiling $\mathfrak{C}=\cup_{g \in G} P_{g}$ and the actions of $G$ on $\mathfrak{C}$ and $M$. In order to distinguish between these actions, we will denote them by $g \cdot x$ and $g(x)$ respectively. Then there is a unique $x \in P_{e}$ such that $z=g \cdot x$. We set $f(z)=x \in P$ and $\varphi(z)=g(x) \in M$. By basic properties of Coxeter groups [2], the mappings $f, \varphi$ are well defined. Moreover, $f: \mathfrak{C} \rightarrow P$ and $\varphi: \mathfrak{C} \rightarrow M$ are the unique $G$-equivariant mappings which are identical on $P_{e} .7$ By construction, both mappings are continuous; they are diffeomorphisms in the interior of each tile, $P_{g} \subset \mathfrak{C}$, and on the interior of the union of any pair of adjacent tiles.

The potential locus of non-differentiability for both $f$ and $\varphi$ is the set $V$ of vertices in the tiling $\mathfrak{C}=\cup_{g \in G} P_{g}$. We have $V=f^{-1}(K(P))$ where $K(P)$ is the set of corners of $P$. By equivariance, $\varphi(V)=$

\footnotetext{
${ }^{7}$ The action of $G$ on $P$ is trivial.
} 
$\cup_{g \in G} g(K(P)) \subset M \&$ There are two kinds of points in $V$ : vertices coming from the corners of $P$ with $\pi$-rational and $\pi$-irrational angles. Their cone angles are integer multiples of $2 \pi$ and are infinite respectively. Vertices $v \in V$ with cone angle $2 \pi$ are, in fact, regular points in $\mathfrak{C}$, and the mappings $f, \varphi$ are both regular there. Around a vertex $v$ with cone angle $2 k \pi>2 \pi$ the mapping $\varphi$ is differentiable, but not a diffeomorphism; it is locally conjugate to $z \mapsto z^{k}$. Near such a vertex, $\varphi$ is a branched covering of degree $k$. At a vertex with infinite cone angle, $\varphi$ has infinite branching.

Remark 3. The set $\varphi(V) \subset M$ is countable. (It is finite iff the group generated by geodesic reflections in the sides of $P$ is a finite Coxeter group. Typically, $\varphi(V) \subset M$ is a dense, countable set.) Let $M=\mathbb{S}^{2}$, and let $z \mapsto z^{\prime}$ denote the antipodal map. Set $F=$ $P \cap\left(\varphi(V) \cup(\varphi(V))^{\prime}\right)$. Points of $F$ are exceptional, in the following sense. Let $z \in P$ be such that the beam $R_{z}$ of billiard orbits emanating from $z$ contains a sub-beam focusing at a corner of $P$. Then $z \in F$. This follows from Proposition 6 below.

Thus, $F$ contains all points $z \in P$ for which the transversality assumption in Condition 2 of section 1.2 fails. Since $F$ is countable, the set of exceptional parameters has measure zero, and Condition $2^{\prime}$ is satisfied. See Remark 1 in section 1 .

Furthermore, the mappings $f$ and $\varphi$ are local isometries. They are isometries on every tile $P_{g} \subset \mathfrak{C}$; we have $f\left(P_{g}\right)=P, \varphi\left(P_{g}\right)=g(P) \subset$ $M$. Let $g \cdot a$ be a side of $P_{g}$, let $h=\sigma_{a} g$ and let $P_{h}$ be the adjacent tile. The maps $f: P_{g} \rightarrow P, P_{h} \rightarrow P$ and $\varphi: P_{g} \rightarrow g(P), P_{h} \rightarrow h(P)$ are coherent around the common (open) side $g \cdot a$. The map $f$ is never an isometry on $P_{g} \cup P_{h}$; for $\varphi$ this is the case iff the interiors of $g(P), h(P)$ are disjoint in $M$. The latter generally fails for nonconvex $P$.

By coherence of $f$ and $\varphi$ across the sides separating adjacent tiles, we lift them to the tangent bundles, obtaining the mappings of unit tangent bundles $F: T \mathfrak{C} \rightarrow T P, \Phi: T \mathfrak{C} \rightarrow T M$, which are also defined on vectors based at the vertices of the tiling $\mathfrak{C}=\cup_{g \in G} P_{g}$. Let $v$ be a vertex, and let $\alpha$ be the angle of the corner $f(v) \in K(P)$. Then $\Phi$ : $T_{v} \mathfrak{C} \rightarrow T_{\varphi(v)} M$ is $m$-to- 1 if $\alpha=m \pi / n$ and $\infty$-to- 1 if $\alpha$ is $\pi$-irrational. The geodesics $\gamma(t)$ in $\mathfrak{C}$ cannot be further extended (generally) once they reach a vertex. All other geodesics in $\mathfrak{C}$ are defined for $-\infty<t<$ $\infty$.

\footnotetext{
8 The representation $M=\cup_{g \in G} g(P)$ is not a tiling, in general.
} 
Using the inclusion $P \subset M$, we identify $T P$ with the subset of $T M$ consisting of $M$-tangent vectors with base-points in $P$, and directed inward. Any $v \in T P$ defines the billiard orbit in $P, \beta(t)=\exp _{P}(t v), 0 \leq$ $t$, and the geodesic in $M, \gamma(t)=\exp _{M}(t v), 0 \leq t$. They are related by the canonical unfolding of billiard orbits. This is an inductive procedure which replaces the consecutive reflections about the sides of $P$ by consecutive reflections of the "latest billiard table" $g(P)$ about the appropriate side, yielding the next billiard table $h(P)$, and continuing the geodesic straight across the common side of $g(P)$ and $h(P)$. See [4] in the planar case and [7, section 3.1, in the general case. Let $x \in P$ and let $v \in T_{x} P$. We denote by $\beta_{v}$ (resp. $\gamma_{v}$ ) the billiard orbit in $P$ (resp. the geodesic in $M$ ) that emanates from $x$ in the direction $v$. The unfolding operator, $U_{x}: \beta_{v} \mapsto \gamma_{v}$, preserves the parametrisations.

Proposition 6. Let $x \in P, v \in T_{x} P$. Identify $P$ and $P_{e} \subset \mathfrak{C}$ and let $x \in P_{e}, v \in T_{x} \mathfrak{C}$ be the corresponding data. Then for $t \in \mathbb{R}_{+}$we have

$$
U_{x}\left(\exp _{P}(v, t)\right)=\varphi\left(\exp _{\mathfrak{C}}(v, t)\right)
$$

Proof. We will freely use the preceding discussion. As $t \in \mathbb{R}_{+}$goes to infinity, $\exp _{P}(v, t)$ runs with the unit speed along a billiard orbit in $P$. The curve $\exp _{\mathfrak{C}}(v, t)$ is the geodesic in $\mathfrak{C}$ defined by the data $(x, v)$, and $\varphi\left(\exp _{\mathfrak{C}}(v, t)\right)$ is the geodesic in $M$ emanating from $x$ in the direction $v$. The billiard orbit in $P$ and the geodesic in $M$ are related by the unfolding operator.

For $x \in P$ let $E_{x} P=T_{x} P \times \mathbb{R}_{+}$be the full tangent space (or the full tangent cone) at $x$. If $S \subset T_{x} P$ is a segment, let $E S_{x} P=S \times \mathbb{R}_{+}$be the corresponding subcone. We use the analogous notation for $x \in \mathfrak{C}$ or $x \in M$. In polar coordinates $(t, \theta)$ in $\mathbb{R}^{2}$ the lebesgue measure on $E_{x} P$ is given by the density $t d t d \theta$.

Corollary 13. Let $x \in P \subset M$ be arbitrary, and let $\exp _{P}^{x}: E_{x} P \rightarrow P$ be the exponential mapping. The pull-back by $\exp _{P}^{x}$ of the lebesgue measure on $P$ to $E_{x} P$ is the smooth measure with the density $d \nu(t, \theta)$.

1. When $M=\mathbb{R}^{2}$, we have $d \nu=t d t d \theta$.

2. When $M=\mathbb{H}^{2}$, we have $d \nu=\sinh t d t d \theta$.

3. When $M=\mathbb{S}^{2}$, we have $d \nu=|\sin t| d t d \theta$.

Proof. By Proposition 6, the measure in question coincides with the pullback to the tangent space $E_{x} M$ of the riemannian measure on $M$ by the exponential map $E_{x} M \rightarrow M$. The latter is well known.

We point out that the preceding material has a billiard map version. We will briefly discuss it now. Let $\beta(t)=(z(t), \theta(t)), t \in \mathbb{R}$, be an orbit 
of the billiard flow. We obtain the corresponding billiard map orbit $\beta_{d}(k), k \in \mathbb{Z}$, by restricting $\beta(t)$ to the consecutive times $t_{k}$ such that $z\left(t_{k}\right) \in \partial P$. The correspondence $\beta(\cdot) \mapsto \beta_{d}(\cdot)$ is invertible. This allows us to formulate the billiard map versions of the universal covering space, the lifting of billiard map orbits to the universal covering space, and the relationship between the liftings and the unfoldings, à là Proposition 6 . Since we are not directly using this material in the body of the paper, we spare the details.

\section{REFERENCES}

[1] J. Cassaigne, P. Hubert and S. Troubetzkoy, Complexity and growth for polygonal billiards, Ann. Inst. Fourier 52 (2002), 835 - 847.

[2] M.W. Davis, Groups generated by reflections and aspherical manifolds not covered by Euclidean space, Ann. Math. 117 (1983), 293 - 324.

[3] G. Galperin, T. Krüger and S. Troubetzkoy, Local instability of orbits in polygonal and polyhedral billiards, Comm. Math. Phys. 169 (1995), 463 - 473.

[4] E. Gutkin, Billiards in polygons, Physica 19 D (1986), 311 - 333.

[5] E. Gutkin, Billiard dynamics: A survey with the emphasis on open problems, Reg. \& Chaot. Dyn. 8 (2003), 1 - 13.

[6] E. Gutkin and N. Haydn, Topological entropy of polygon exchange transformations and polygonal billiards, Erg. Theo. \& Dyn. Syst. 17 (1997), 849 867.

[7] E. Gutkin and S. Tabachnikov, Complexity of piecewise convex transformations in two dimensions, with applications to polygonal billiards on surfaces of constant curvature, Moscow Math. J. 6 (2006), 673 - 701.

[8] E. Gutkin and S. Troubetzkoy, Directional flows and strong recurrence for polygonal billiards, Pitman Res. Not. Math. 362 (1996), 21 - 45.

[9] P. Hubert, Complexité de suites définies par des billards rationnels, Bull. Soc. Math. Fr. 123 (1995), 257 - 270.

[10] A. Katok and B. Hasselblatt, Introduction to the Modern Theory of Dynamical Systems, Cambridge University Press, Cambridge 1995.

[11] H. Masur, The growth rate of trajectories of a quadratic differential, Erg. Theo. \& Dyn. Syst. 10 (1990), $151-176$.

imPA, Rio de Janeiro, Brasil and UMK, Torun, Poland; IMPAN, WARSzaWa, Poland

E-mail address: gutkin@impa.br, gutkin@mat.uni.torun.pl;rams@impan.gov.pl 